\title{
Comparison of the Growth Performance of Nannochloropsis oceanica IMET1 and Nannochloropsis gaditana CCMP526 under Various Culture Conditions
}

\author{
Yongxue Chi, Yasuyuki Takiguchi* \\ Department of Life and Environmental Sciences, Chiba Institute of Technology, Chiba, Japan
}

Email address:

yongxue101@hotmail.com (Yongxue Chi)

To cite this article:

Yongxue Chi, Yasuyuki Takiguchi. Comparison of the Growth Performance of Nannochloropsis oceanica IMET1 and Nannochloropsis gaditana CCMP526 under Various Culture Conditions. Journal of Plant Sciences. Vol. 3, No. 1, 2015, pp. 9-13. doi: $10.11648 /$ j.jps.20150301.12

\begin{abstract}
We studied the growth performance of Nannochloropsis oceanica IMET1 under various culture conditions, including different $\mathrm{CO}_{2}$ concentrations, temperature, or light intensities compared with that of $N$. gaditana CCMP526. When $\mathrm{CO}_{2}$ concentrations were changed, the growth rates of $N$. oceanica IMET1 and $N$. gaditana CCMP526 were the highest at a $\mathrm{CO}_{2}$ concentration of $2 \mathrm{vol} \%$. N. oceanica IMET1 had a higher overall growth rate than that of $N$. gaditana CCMP526. The best growth rate occurred at $30^{\circ} \mathrm{C}$, and higher growth rates were generally exhibited by $N$. oceanica IMET1 than $N$. gaditana CCMP526. The growth rate of $N$. oceanica IMET1 was higher than that of $N$. gaditana CCMP526 when light intensity was changed. These results indicate that $N$. oceanica IMET1 has better growth performance compared with that of $N$. gaditana CCMP526. The optimum growth conditions for $N$. oceanica IMET1 is $\mathrm{CO}_{2}$ concentration of 2 vol\%, light intensity of 53 $\mu \mathrm{mol} / \mathrm{m}^{2} / \mathrm{s}$, and $30^{\circ} \mathrm{C}$ in modified BG11 medium.
\end{abstract}

Keywords: Nannochloropsis oceanica IMET1, N. gaditana CCMP526, Growth Performance,

Culture Conditions, Microalgae

\section{Introduction}

Nannocholopsis sp., which includes marine, fresh, and brackish water algal species, is a potential resource for biofuel production [1-5]. In addition, Nannocholopsis sp. is a resource for high quality protein [6], eicosapentaenoic acid [4], and omega-3 fatty acid [7].

Nannochloropsis oceanica IMET1 collected from Eilat, Israel has been maintained at the Institute of Marine and Environmental Technology (IMET) for $>10$ years. N.oceanica IMET1 has also been distributed to and studied in other laboratories.

Wang et al. investigated the effect of bacterial communities isolated from groundwater at different temperatures on biofuel production using $N$. oceanica IMET1 [8]. Xiao et al. evaluated the effects of nitrogen deficiency stress on liquid content, fatty acid distribution, and metabolic profiles under $\mathrm{CO}_{2}$ concentrations of $1.5-2 \mathrm{vol} \%$ at $20^{\circ} \mathrm{C}$ [9]. Dong et al. reported that long-term nitrate depletion changes the $N$. oceanica IMET1 proteome using a two-dimensional gel electrophoresis proteomic approach [1]. Ma et al. applied heavy-ion irradiation to improve $N$. oceanica IMET1 characteristics for biofuel production [10]. $\mathrm{Li}$ et al. clarified the triacylglycerol-producing mechanism in $N$. oceanica IMET1 under N-repleted and N-depleted conditions [11].

The growth performance and characteristics of IMET1 under various culture conditions such as different temperatures, $\mathrm{CO}_{2}$ concentrations, light intensities, and media have not been reported. Understanding growth performance under different conditions is important to determine the optimum conditions for producing biofuel, eicosapentaenoic acid, and omega-3 fatty acid. Therefore, we investigated the effects of $\mathrm{CO}_{2}$ concentration, temperature, light intensity, and culture media on the growth performance of $N$. oceanicaIMET1 and N. gaditana CCMP526.

\section{Materials and Methods}

\subsection{Algal Strains and Culture Conditions}

$N$. oceanicaIMET1 and $N$. gaditanaCCMP526 were used 
in this study. $N$. oceanica IMET1 was obtained from the IMET, Maryland University Center for Environmental Science. We used N. gaditanaCCMP526 based on the good growth performance of other CCMP under the same culture conditions. $N$. gaditana CCMP526 was purchased from the National Center for Marine Microalgae and Microbiota.

A modified BG11 (MBG11) medium and artificial seawater (ASW) medium were used to grow both strains.

BG11 [12] is a common culture medium for freshwater algae and contains more nutrients than those in ASW medium. MBG11 medium is BG11 with salinity adjusted to $30 \mathrm{ppm}$ with $\mathrm{NaCl}$. ASW medium was prepared according to the ingredients described by Radakovits et al. [13].

Both $N$. oceanica IMET1 and $N$. gaditana CCMP526 were routinely maintained in flasks containing ASW. The log-phase cultures were harvested, and the pellets were washed $(6,000 \times \mathrm{g}, 10 \mathrm{~min})$ with the respective medium to remove wastes. The algal pellets were re-suspended in either ASW or MBG11 medium. One ml of each culture was added to one well of a 48-well Costar plate (Corning Glass, Corning, NY, USA), and six replicates were performed for each culture. The microplates containing the algal cultures were placed inside a GasPak ${ }^{\mathrm{TM}}$ bag (Becton Dickinson, Parsippany, NJ, USA).

\subsection{Specific Growth Rate}

Cell density (O.D. ${ }_{600}$ ) was determined at an absorbance of $600 \mathrm{~nm}$ using a multi-mode microplate reader (Spectra Max M5; Molecular Devices, Sunnyvale, CA, USA). The microplate was taken from the $\mathrm{GasPak}^{\mathrm{TM}}$ bag to read absorbance and was then placed back into the $\mathrm{GasPak}^{\mathrm{TM}}$ bag.

Cell density on day 0 was set to an O.D.600 of 0.35 . Cell density was measured every 2 days at the media change time. All O.D. ${ }_{600}$ data indicated the average value of $n=6$ with the standard deviation.

Specific growth rate $\mu$ was calculated from the O.D. 600 value after 2 days of culture $\left(\mathrm{OD}_{2}\right)$ and the O.D. ${ }_{600}$ value after 8 days of culture $\left(\mathrm{OD}_{8}\right)$ using the following equation

$$
\mu=\ln \left(O D_{8} / O D_{2}\right) / 6
$$

The specific growth rate was the average value of $n=6$ with the standard deviation.

\subsection{Growth Conditions}

The effect of $\mathrm{CO}_{2}$ concentration on growth rate was investigated under the following conditions. The $\mathrm{CO}_{2}$ concentration (vol\%) was set by blending pure $\mathrm{CO}_{2}$ and air using a gas mixture device with two gas flow meters [14]. The microplates containing the algal cultures were placed in a GasPak ${ }^{\mathrm{TM}}$ bag and charged with $0,2,10,15$, and $20 \mathrm{vol} \%$. The algal cultures were incubated at $23^{\circ} \mathrm{C}$ with a light intensity of $53 \mu \mathrm{mol} / \mathrm{m}^{2} / \mathrm{s}$ and a $12 \mathrm{~h} / 12 \mathrm{~h}$ light-dark cycle.

The effect of light intensity on growth rate was investigated under the following conditions. Light intensities were set to $13,53,82$, and $132 \mu \mathrm{mol} / \mathrm{m}^{2} / \mathrm{s}$, respectively. The algal cultures in the microplates were incubated under a $\mathrm{CO}_{2}$ concentration of $2 \mathrm{vol} \%$ at $23^{\circ} \mathrm{C}$ with a $12 \mathrm{~h} / 12 \mathrm{~h}$ light-dark cycle. Each treatment contained six replicates.

The effect of temperature on growth rate was investigated under the following conditions. Temperature was set to 10 , 23 , and $30^{\circ} \mathrm{C}$, respectively. The algal cultures in the microplates were incubated under a 2 vol $\% \mathrm{CO}_{2}$ concentration, light intensity of $53 \mu \mathrm{mol} / \mathrm{m}^{2} / \mathrm{s}$, and a $12 \mathrm{~h} / 12$ $\mathrm{h}$ light-dark cycle. Each treatment contained six replicates.

\section{Results and Discussion}

\subsection{Growth Performance under Different $\mathrm{CO}_{2}$ Concentrations}

Fig. 1 shows the culture time and O.D.600 values for $N$. oceanica IMET1 and $N$. gaditana CCMP526 in ASW or MBG11 under various $\mathrm{CO}_{2}$ concentrations. Fig. 2 shows the comparison of growth rate in ASW or MBG11 under various $\mathrm{CO}_{2}$ concentrations.

The growth rates of $N$. oceanica IMET1 and $N$. gaditana CCMP526 were the highest at a $\mathrm{CO}_{2}$ concentration of $2 \mathrm{vol} \%$. Moreover, the growth rates of $N$. oceanica IMET1 and $N$. gaditana CCMP526 were higher in MBG11 medium than those in ASW medium. Growth of both strains was inhibited at higher $\mathrm{CO}_{2}$ concentrations (Figs. 1, 2).

$N$. oceanica IMET1 maintained relatively higher growth rates compared with those of $N$. gaditana CCMP526 at $\mathrm{CO}_{2}$ concentrations of 10,15 , and $20 \mathrm{vol} \%$. The growth rate of $N$. oceanica IMET1 outperformed the growth rate of $N$. gaditana CCMP526 in both ASW and MBG11 culture media at the higher $\mathrm{CO}_{2}$ concentrations. At a $\mathrm{CO}_{2}$ concentration of $20 \mathrm{vol} \%$, in MBG11 medium, $N$. gaditanaCCMP526 nearly ceased growing $\left(\mu=0.002 \mathrm{~d}^{-1}\right)$; however, the growth of $N$. oceanica IMET1 only slowed down at $\sim 35$ vol\% compared to the highest growth rate at the 2 vol\% $\mathrm{CO}_{2}$ concentration. Both strains seemed to grow better in MBG11 medium than in ASW medium, particularly under the higher $\mathrm{CO}_{2}$ concentrations. The growth rate of $N$. oceanica IMET1 outperformed that of $N$. gaditana CCMP526 at all tested $\mathrm{CO}_{2}$ concentrations in ASW medium.

$\mathrm{Hu}$ and $\mathrm{Gao}$ reported that biomass yield while supplying air with $2,800 \mu \mathrm{l} \mathrm{CO}_{2} \mathrm{l}^{-1}$ is higher than that supplying air with 350 $\mu \mathrm{l} \mathrm{CO}_{2} \mathrm{l}^{-1}$, when Nannochloropsis sp. (PP983) is used [15]. Chiu et al. reported that biomass concentration and growth rate at a $\mathrm{CO}_{2}$ concentration of $2 \mathrm{vol} \%$ were higher than those at other $\mathrm{CO}_{2}$ concentrations because $N$. oculata utilizes $\mathrm{CO}_{2}$ in response to adding $\mathrm{CO}_{2}$ [16]. We also found the highest growth rate at a $\mathrm{CO}_{2}$ concentration of $2 \mathrm{vol} \%$. Chiu et al. also reported that biomass concentration and growth rate decreased when the $\mathrm{CO}_{2}$ concentration was $>5 \mathrm{vol} \%$, which correlates with our result. Although growth rates of $N$. oceanica IMET1 and $N$. gaditana CCMP526 decreased when $\mathrm{CO}_{2}$ concentration was $>10 \mathrm{vol} \%$, the growth rate of $N$. oceanica IMET1 was higher than that of $N$. gaditana CCMP526. The growth performance of Nannochloropsis spp. was not clear; therefore, it is difficult to understand the reasons for the difference in growth rate over $\mathrm{CO}_{2}$ concentration of $10 \mathrm{vol} \%$ between $N$. oceanica IMET 1 and 
$N$. gaditana CCMP526. This may have resulted from the different genetic backgrounds between $N$. oceanica IMET1 and N. gaditana CCMP526, which are not closely related [13]. In addition, $N$. oceanicaIMET1 has different genes in the Kennedy pathway from those of $C$. reinhardtii [11].
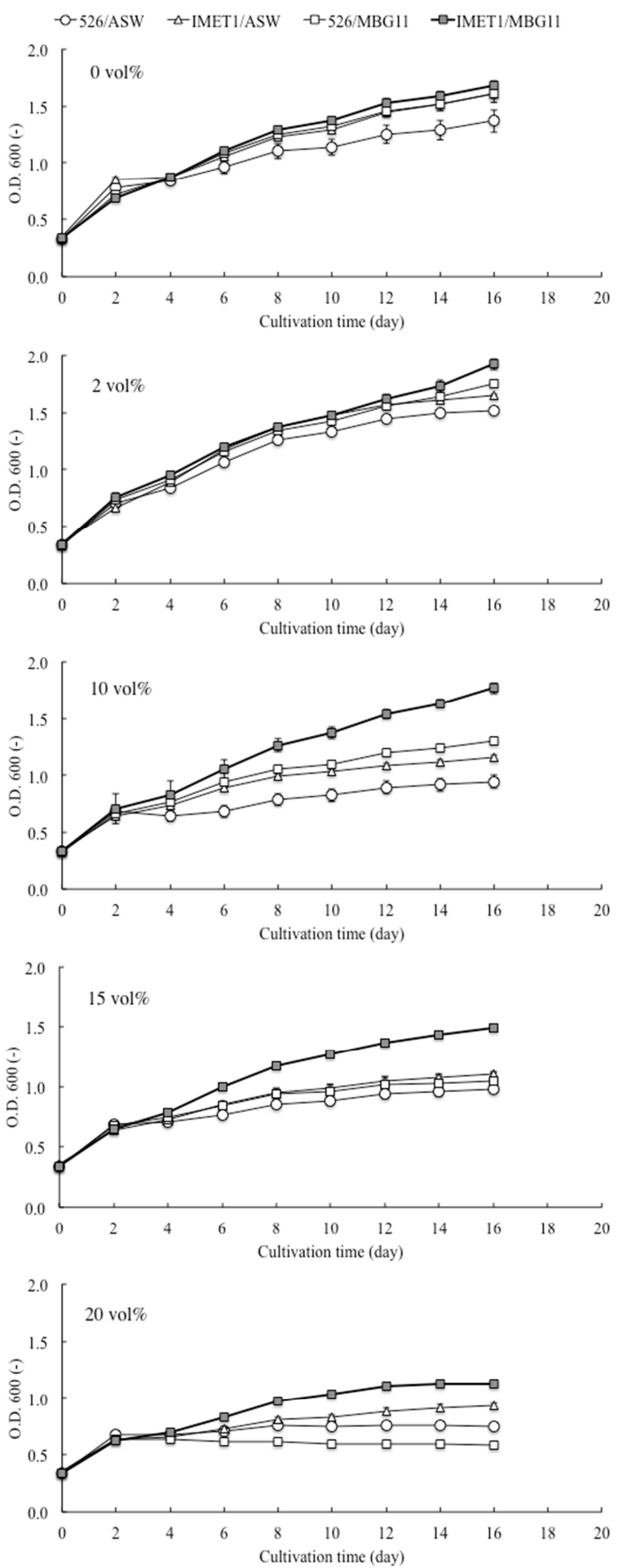

Figure 1. Growth curves for Nannochloropsis gaditana CCMP526 and N. oceanica IMET1 cultured at $\mathrm{CO}_{2}$ concentrations of $0,2,10,15$, and $20 \mathrm{vol} \%$ All cultures were maintained at $23^{\circ} \mathrm{C}$ with $53 \mu \mathrm{mol} / \mathrm{m}^{2} / \mathrm{s}$ light intensity and a $12 \mathrm{~h} / 12 \mathrm{~h}$ light/dark (LED source) cycle. The two culture media (MBG11 and $A S W)$ were compared. Each data point is the average of six replicates $(n=6)$, and error bars represent standard deviations $(n=6), p<0.05$.

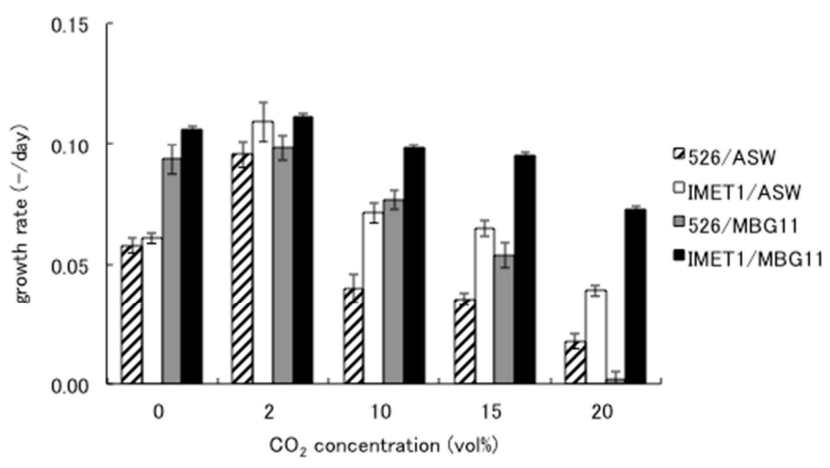

Figure 2. Specific growth rates of Nannochloropsis oceanica IMET1 and N. gaditana CCMP526 under different $\mathrm{CO}_{2}$ concentrations. Both strains were cultured at $23^{\circ} \mathrm{C}$ with a light intensity of $53 \mu \mathrm{mol} / \mathrm{m}^{2} / \mathrm{s}$ and a $12 \mathrm{~h} / 12 \mathrm{~h}$ light/dark (LED source) cycle. Each data point is the average of six replicates, and error bars represent standard deviations $(n=6), p<0.05$.

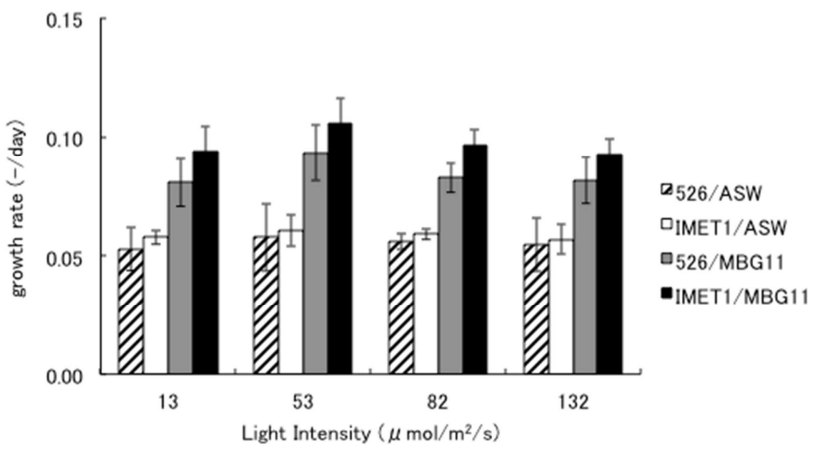

Figure 3. Specific growth rates of Nannochloropsis oceanica IMET1 and N. gaditana CCMP526, cultured under different light intensities (13, 53, 82, and $133 \mu \mathrm{mol} / \mathrm{m}^{2} / \mathrm{s}$ ), at $23^{\circ} \mathrm{C}$ with a $12 \mathrm{~h} / 12 \mathrm{~h}$ light/dark cycle. Each data point is the average of six replicates, and error bars represent standard deviations $(n=6), p<0.05$.

\subsection{Growth Performance under Four Light Intensities}

Fig. 3 shows the comparison of growth rates between $N$. oceanica IMET1 and $N$. gaditana CCMP526 irradiated under light intensities of $13,53,82$, and $132 \mu \mathrm{mol} / \mathrm{m}^{2} / \mathrm{s}$. The growth rate of $N$. oceanica IMET1 in ASW was nearly the same as the growth rate of $N$. gaditana CCMP526 in ASW at the various light intensities. The growth rates of $N$. oceanica IMET1 and $N$. gaditana CCMP526 in MBG11 medium were higher than those in ASW. The growth rate of $N$. oceanica IMET1 in MBG11 was maximum at a light intensity of 53 $\mu \mathrm{mol} / \mathrm{m}^{2} / \mathrm{s}$ and was higher than that of $N$. gaditana CCMP526 regardless of light intensity. These results indicate that light intensity affects the growth rate of Nannochloropsis in MBG11. In addition, the growth rate of $N$. oceanica IMET1 in MBG11 was the highest at a light intensity of 53 $\mu \mathrm{mol} / \mathrm{m}^{2} / \mathrm{s}$.

Optimal growth of both strains occurred at $53 \mu \mathrm{mol} / \mathrm{m}^{2} / \mathrm{s}$. Because the experiments were conducted in 48-well microplates, the optimal light intensity was generally lower than the optimal light intensity of a flask-based culture system. Growth of both $N$. oceanica IMET1 and $N$. gaditana CCMP526 was photoinhibited to some extent at a light intensity of $132 \mu \mathrm{mol} / \mathrm{m}^{2} / \mathrm{s}$. N. oceanica IMET1 and $N$. 
gaditana CCMP526 again preferred MBG11 medium to ASW medium. Dipasmita also reported the same result in a light intensity experiment in which the growth rate of Nannochloropsis sp. declined under high light intensity in 13 $\mathrm{g} / \mathrm{L} \mathrm{NaCl}[18]$.

\subsection{Growth Performance under Different Temperatures}

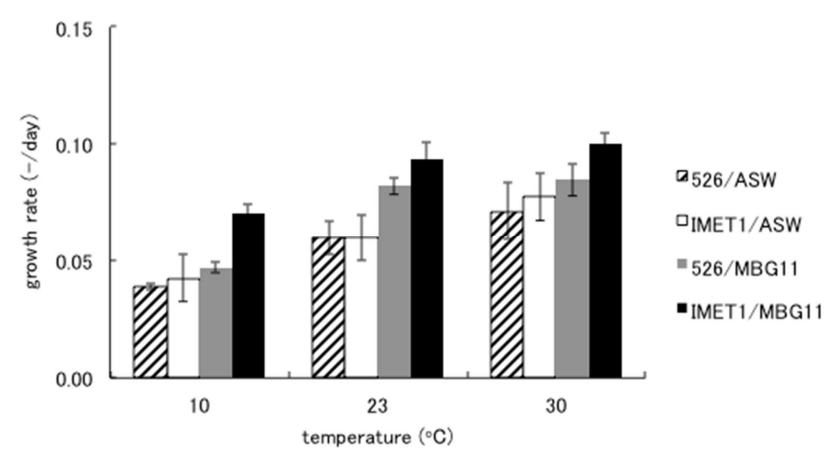

Figure 4. Specific growth rates of Nannochloropsis oceanica IMET1 and N. gaditana CCMP526 under different temperatures $\left(10,23\right.$, and $\left.30^{\circ} \mathrm{C}\right)$. Both strains were cultured at a light intensity of $53 \mu \mathrm{mol} / \mathrm{m}^{2} / \mathrm{s}$ with a $12 \mathrm{~h} / 12 \mathrm{~h}$ light/dark (LED source) cycle. Each data point is the average of six replicates, and error bars represent standard deviations $(n=6), p<0.05$.

Fig. 4 shows the comparison of the growth rates between $N$. oceanica IMET1 and N. gaditana CCMP526 at 10, 23, and $30^{\circ} \mathrm{C}$. The growth rates of $N$. oceanica IMET1 and $N$. gaditana CCMP526 increased with a rise in temperature. The growth rate of $N$. oceanica IMET1 was higher than that of $N$. gaditana CCMP526 at all temperatures, regardless of the medium. These results indicate that the growth rate of $N$. oceanica IMET1 at $30^{\circ} \mathrm{C}$ was the best in MBG11 medium ( $\mu$ $=0.1 \mathrm{~d}^{-1}$ ). Growth of $N$. oceanica IMET1 was inhibited at $10^{\circ} \mathrm{C}$ in a $\mathrm{CO}_{2}$ concentration of $\sim 25 \mathrm{vol} \%$. Although the growth rates of $N$. gaditana CCMP526 at 23 and $30^{\circ} \mathrm{C}$ were acceptable, the growth rate of $N$. gaditana CCMP526 at $10^{\circ} \mathrm{C}$ was inhibited $\left(\mu=0.03 \mathrm{~d}^{-1}\right)$. N. oceanicaIMET1 and $\mathrm{N}$. gaditanaCCMP526 grew better in MBG11 medium than that in ASW medium. In general, both strains maintained rapid growth at 23 and $30^{\circ} \mathrm{C}$; however, their growth rates significantly decreased when temperature dropped to $10^{\circ} \mathrm{C}$. $\mathrm{Wu}$ et al. reported that the maximum growth rate of Monoraphidium sp. SB2 occurred at $30^{\circ} \mathrm{C}$ [18]. Their result correlated with our result. These results suggest that $N$. oceanica IMET1 had higher growth rates than those of $N$. gaditana CCMP526, regardless of temperature or culture conditions. These results may be because of the activities of five putative genes related to carbon fixation such as carbonic anhydrate mediated carbon-concentrating metabolism and a $\mathrm{C} 4$ cycle mechanism in $N$. oceanica IMET1 [11].

In this study, the growth performance of $N$. oceanica IMET1 and $N$. gaditana CCMP526 was compared in 48-well microplates. The microplate culture system is different from other culture systems such as flasks or bioreactors. Therefore, it may be difficult to compare our results with those obtained from large cultivation systems with air bubbling. For example, growth of $N$. oculataNCTU-3 was completely inhibited when cultured at $\mathrm{CO}_{2}$ concentrations of $5 \mathrm{vol} \%, 10$ vol $\%$, and 15 vol $\%$ in flasks [16]. In our experiments, different concentrations of $\mathrm{CO}_{2}$ were contained in $\mathrm{GasPak}^{\mathrm{TM}}$ bags. Nevertheless, the growth rates of these two Nannochloropsis strains were compared in parallel in this study and they differed greatly in terms of their capability of handling different environmental stressors.

\section{Conclusion}

We investigated the growth performance of $N$. oceanica IMET1 compared with that of $N$. gaditana CCMP526. The growth performance of $N$. oceanica IMET1 was better than that of $N$. gaditana CCMP526. In particular, the growth rate of $N$. oceanica IMET1 was higher than that of $N$. gaditana CCMP526, regardless of culture medium under $\mathrm{CO}_{2}$ concentrations $>10 \mathrm{vol} \%$. In addition, growth of $N$. oceanica IMET1 was maintained at a high level under a wide range of light intensities and temperatures in both culture media. The optimum growing conditions for $\mathrm{N}$. oceanica IMET1 were a $\mathrm{CO}_{2}$ concentration of $2 \mathrm{vol} \%$, light intensity of $53 \mu \mathrm{mol} / \mathrm{m}^{2} / \mathrm{s}$, and $30^{\circ} \mathrm{C}$ when MBG11 was used.

\section{Acknowledgment}

We acknowledge support from the Maryland Industrial Partnership Program and support from Dr. Feng Chen, and appreciate Professor Kaoru Onoe for supporting the editing of the manuscript.

\section{References}

[1] H. P. Dong, E. Williams, D. Z. Wang, Z. X. Xi, R. C. Hsia, A. Jenck, R. Halden, J. Li, F. Chen, R. P. Allen, "Responses of Nannochloropsis oceanica IMET1 to long-term nitrogen starvation and recovery," Plant Physiol, vol. 162, pp. 1110-1126, June 2013.

[2] O. Kilian, C. S. E. Benemann, K. K. Niyogi, B. Vick, "High-efficiency homologous recombination in the oil-producing alga Nannochloropsis sp.," Proc. Natl. Acad. Sci, vol. 108, pp. 21265-21269, December 2011.

[3] A. Sukenik, O. Zamora, Y. Carmeli, "Biochemical quality of marine unicellular algae with emphasis on lipid composition. II. Nannochloropsis sp.," Aquaculture, vol. 117, pp. 313-326, November 1993.

[4] A. Sukenik, Y. Carmeli, T. Berner, "Regulation of fatty acid composition by irradiance level in the eustigmatophyte Nannochloropsis sp.," J. Phycol, vol. 25, pp. 686-692, December 1989.

[5] Y. Suen, J. S. Hubbard, G. Holzer, T. G. Tornabene, "Total lipid production of the green alga Nannochloropsis sp. QII under different nitrogen regimes," J. Phycol, vol. 23, pp. 289-296, June 1987.

[6] J. K. Volkman, M. R. Brown, G. A. Dunstan, S. W. Jeffrey, "The biochemical composition of marine microalgae from the class Eustigmatophyceae," J. Phycol, vol. 29, pp. 69-78, February 1993. 
[7] J. H. Lee, J. H. O'Keefe, C. J. Lavie, W. S. Harris, "Omega-3 fatty acid: Cardiovascular benefits, sources and sustainability," Nat. Rev. Cardiol, vol. 6, pp. 753-758, December 2009.

[8] H. Wang, H. D. Laughinghouse 4th, Anderson M.A., Chen F., Williams E., Place A. R., Zmora O., Zohar Y.,Zheng T., HillR.T., "Novel Bacterial isolate from Permian groundwater, capable of aggregation potential biofuel-producting microalge Nannochloropsis oceanica IMET1," Appl. Environ. Microbiol, vol. 78, pp. 1445-1453, March 2012.

[9] Y. Xiao, J. Zhang, J. Cui, Y. Feng, Q. Cui, "Metabolic profiles of Nannochloropsis oceanica IMET1 under nitrogen-deficiency stress," Bioresour. Technol, vol. 130, pp. 731-738, February 2013.

[10] Y. Ma, Z. Wang, M. Zhu, C. Yu, Y. Cao, D. Zhang, G. Zhou, "Increased liquid productivity and TGA content in Nannochloropsis by heavy-ion irradiation mutagenesis," Bioresour. Technol, vol. 136, pp. 360-367, 2013.

[11] J. Li, D. Han, D. Wang, K. Ning, J. Jia, L. Wei, X. Jing, S. Huang, J. Chen, Y. Li, Q. Hu, J. Xu, "Choreography of transcriptomes and lipidomes of Nannochloropsis reveals the mechanisms of oil synthesis in microalge," Plant Cell, vol. 26, pp. 1645-1665, April 2014.

[12] R. Y. Stanier, R. Kunisawa, M. Mandel, G. Cohen, "Purification and properties of unicellular blue-green algae (order Chroococcales)," Bacteriol. Rev, vol. 35, pp. 171-205, June 1971.
[13] R. Radakovits, R. E. Jinkerson, S. I. Fuerstenberg, H. Tae, R. E. Settlage, J. L. Boore, M. C. Posewitz, "Draft genome sequence and genetic transformation of the oleaginous alga Nannochloropsis gaditana," Nat. Commun, vol. 3, pp. 686-695, February 2012.

[14] Z. Liu, F. Zhang, F. Chen, "High throughput screening of $\mathrm{CO}_{2}$-tolerating microalgae using GasPak bags," Aquat. Biosyst, vol. 9, pp. 23, December 2013.

[15] H. Hu, K. Gao, "Optimization of growth and fatty acid composition of a unicellular marine picoplankton, Nannochloropsis sp., with enriched carbon sources," Biotechnol. Lett, vol. 25, pp. 421-425, March 2003.

[16] S. Y. Chiu, C. Y. Kao, M. T. Tsai, S. C. Ong, C. H. Chen, C. S. Lin, "Lipid accumulation and $\mathrm{CO}_{2}$ utilization of Nannochloropsis oculata in response to $\mathrm{CO}_{2}$ aeration," Bioresour. Technol, vol. 8, pp. 833-838, January 2009.

[17] P. Dipasmita, K. G. Inna, C. Zvi, B. Sammy, "The effect of light, salinity, and nitrogen availability on lipid production by Nannochloropsissp.," Appl. Microbiol. Biotechnol., vol. 90, pp. 1429-1441, May 2011.

[18] L. F. Wu, P. C. Chen, C. M. Lee, "The effects of nitrogen sources and temperature on cell growth and lipid accumulation of microalgae," Int. Biodeter. Biodegr, vol. 85, pp. 506-510, November 2013. 